\title{
Method of Calculation the Accessibility of the Information and Telecommunication Networks of the Tactical Link of Management
}

\author{
Leonid Bondarenko \\ Military Institute of Telecommunications and Information Technologies, Named after the Heroes of Krut, \\ Kyiv,01001,Ukraine \\ E-mail: lablab50@ukr.net \\ Mykola Masesov \\ Military Institute of Telecommunications and Information Technologies, Named after the Heroes of Krut, Kyiv, 01001, \\ Ukraine \\ E-mail:masesov@ukr.net
}

\section{Katerina Buzayeva}

Military Institute of Telecommunications and Information Technologies, Named after the Heroes of Krut, Kyiv,01001,Ukraine

E-mail: ggggggryui@gmail.com

\section{Iryna Hurzhii}

Military Institute of Telecommunications and Information Technologies, Named after the Heroes of Krut, Kyiv,01001,Ukraine

E-mail: irisha.kiev@gmail.com

\section{Oksana Ilienko}

Military Institute of Telecommunications and Information Technologies, Named after the Heroes of Krut, Kyiv,01001,Ukraine

E-mail: oks.ilenko7@gmail.com

\author{
Viktor Gnatyuk \\ National Aviation University, Kyiv,03058, Ukraine \\ E-mail: viktorgnatyuk@ukr.net
}

Received: 20 January 2021; Accepted: 20 April 2021; Published: 08 October 2021

\begin{abstract}
The article reflects the views of the authors on the method for the operational calculation of the indicator of the functioning of special-purpose information and telecommunication networks of the tactical level of control according to the criterion of "network availability". Improvement of weapons and military equipment, forms and methods of combat, as well as a change in views on command and control, put forward ever more stringent requirements for the information and telecommunication system in general and for its elements in particular. The problems of development, planning of combat use and operation of information and telecommunication networks of the tactical control level are their heterogeneity, since they use heterogeneous transmission systems (radio and wire) together. The characteristic features of the planning, deployment and operation of information and telecommunication networks of the tactical control level are a high degree of uncertainty in the characteristics of their functioning and the lack of initial data in planning, which necessitates the improvement of the methodological basis for conducting operational calculations. Based on the analysis of ITU-T recommendations, scientific publications and the practice of combat use of the defense forces, it becomes clear that the operational calculation of individual criteria and indicators of the functioning of information and telecommunication systems of the tactical control level needs to be improved, since these systems have their own characteristic features. The material presented in the article makes it possible to develop new approaches to solving the problem of a reasonable calculation of the required bandwidth of the access node of the information and telecommunications network of the tactical control link. The significance and value of this study lies in the fact that in the conditions of continuous improvement of the forms and methods of warfare, high dynamism of changes in the states of information and telecommunication systems of the tactical control level, the correct choice of
\end{abstract}


the apparatus for assessing the decisions made should play a decisive role in shortening the deployment planning cycle and sustainable functioning of the system management.

Index Terms: Bandwidth ability, access node, network performance, telecommunications network, tactical link.

\section{Introduction}

Recently, in many military scientific publications, more and more publications have begun to appear on the topic of forms and methods of warfare with the aim of gaining a military advantage. Their analysis shows that at present the opinions of various authors are very contradictory and differ significantly from each other. Some argue that success in combat depends on the ability of commanders to logically build an operational model of future combat, while others argue that success depends on actions taken at the level of tactical control as a result of dynamic changes in information and telecommunications systems. When carrying out this study, it is necessary to take into account that the basis of the communication system of groupings of troops is information and telecommunication networks for special purposes of the tactical control level, the functioning of which will be carried out in rather difficult and unfavorable environmental conditions, which requires their stable functioning in real time.

In the context of growing information needs, the significance of individual properties of the control system that characterize information and telecommunication networks has changed significantly, while the properties themselves have significantly transformed.

Making a decision to build a communication system of any level, as a rule, includes determining the purpose of its functioning, choosing and calculating indicators with substantiation of assessment criteria, synthesizing alternative structures and finding the best option.

The most characteristic features of the deployment of information and telecommunication systems at the tactical level of control are the lack of initial data during planning and a high degree of uncertainty in their functioning. In such conditions, it is important to choose the correct apparatus for evaluating the decisions made, which will allow officials of the communications management bodies to gain confidence in their actions and results, the results achieved.

Since information and telecommunication systems have many properties, the list of properties by which it is advisable to assess the quality of systems should be limited. In practice, this means that quality assessment should be based not on all, but only on the most significant properties, reflecting the main aspects of information and telecommunication systems and their individual elements. This approach makes it possible to focus on the main characteristics of information and telecommunication systems, which allow them to solve their main task in the predictable operating conditions in the best way. Therefore, a comprehensive comprehensive and objective assessment of the effectiveness of information and telecommunication systems can be carried out using a system of indicators and criteria, including the main and additional indicators for the main and particular properties, respectively.

\section{Motivation behind this Work}

The practice of the troops has convincingly shown that in the theory and practice of organizing communications there is a need to improve the methodological approaches to calculating the indicators of information and telecommunication systems by choosing a mathematical apparatus that will correspond to the modern conditions of the functioning of control systems and provide a more adequate assessment of the effectiveness of information and telecommunication systems operating or unfold.

The purpose of the research of the development lies in the fact that in the conditions of constant improvement of the forms and methods of command actions, the high dynamism of changes in the states of information and telecommunication systems of the tactical control level, due to the nature of command actions, as well as changes in approaches to planning the combat employment of troops, the correct choice of the apparatus for assessing the adopted solutions that will enable communications officials to gain confidence in their actions and the results to be achieved.

The article proposes a methodological basis for the operational calculation of the key indicator "availability" of ITN of the tactical control level and to develop a method of operational calculation that would allow officials of communications control bodies to make high-quality operational calculations of network performance indicators in a short time, taking into account the specifics of their purpose and construction.

\section{Related Work}

Quite a lot of works and scientific articles are devoted to the analysis of the functioning of information and telecommunication networks, each of which considers certain indicators of the system and their dependence on other particular indicators ITU -T Y.1540 Recommendation [1] provides a rationale for defining an IP service availability function (from a network operator's point of view) that establishes sufficient availability in terms of packet loss. Therefore, an assessment of condition based on losses only and criteria previously agreed upon for assessment of 
condition (5 minutes, $75 \%$ loss) are considered sufficient

In the article of Yanovsky G.G. [2] provides an overview of methods for maintaining quality of service in networks built based on IP-based protocols. The ITU-T Recommendations Y.1540 and Y.1541, which define network characteristics and quality of service standards for IP networks. Based on the analysis of methods for maintaining the quality of service in IP networks, it was concluded that the standards of quality of service have been achieved on the basis of providing guaranteed bandwidth, increasing the efficiency of network devices, which is a prerequisite for achieving the final goal of planning the operation of ITN.

An ITU-R F.2058 report [3] describes the design issues of fixed wireless communication systems used in access networks. The report proposes a methodology for calculating effective bandwidth for different types of services by classifying them according to several attributes (delay requirement or priority requirement) and applying one appropriate equation to calculate effective bandwidth, refers to it and indirectly expresses the service requirement for QoS. Such proposals are fully consistent with the objectives of the study in solving the problems of carrying out operational calculations of the performance indicators of special purpose ITN.

In works $[4,5]$, a method is proposed for determining the effectiveness of a communication system, which allows you to model its structure. The proposed approach consists in the use of a generalized indicator, which allows assessing the communication system taking into account the influence of heterogeneous factors on it, complex accounting of throughput, stability and availability - spatial and information-channel. The approach proposed in the works quite fully corresponds to the objectives of the research carried out in solving the problems of carrying out operational calculations of planning, deployment and operation of ITN of the tactical control level, but does not contain the methodology of operational calculations.

The article [6] proposes a mathematical formulation and solution of the problem of synthesizing the topology of a multiservice telecommunication system, built according to the NGN concept, on a section of an access aggregation network. As a criterion for assessing the compliance of the access network with the requirements, the indicator of the operator's maximum profit was chosen.

The article [7] describes the procedure for solving the problem of synthesizing the topology of the access network of a telecommunications system according to the criterion of maximum operator profit in the absence and presence of additional requirements for connectivity. In these articles [7, 8], the calculations are focused on network operators with the goal of making a profit in their activities while ensuring a given level of service quality. Such approaches are based on deterministic indicators of the quality of ITN functioning, the use of complex mathematical calculations and in essence do not take into account the peculiarities of the functioning of special-purpose ITN.

The solution to the problem is based on the use of a mathematical model of a multilayer graph. In the article by A.G. Korkin [8] provides an overview of the types of hybrid automatic retransmission request, which are used in modern technologies of wireless access networks. A technique for estimating the bandwidth ability when implementing a hybrid automatic retransmission request is proposed. The technique is of a research nature, contains a complex mathematical apparatus, and is based on calculating the probabilistic-temporal characteristics of a typical radio access system of the IEEE 802.16e standard using adaptive modulation and coding schemes for rate control. The proposed methodology does not correspond to the objectives of the study in solving the problems of carrying out operational calculations.

The article by A.V. Golubintsev [9] present some aspects of the study of the problem of the bandwidth of the systems of broadband wireless subscriber access of cellular networks and the methods of its calculation are considered. The technique is of a research nature, contains a complex mathematical apparatus and a set of input data for calculations, which are tied to the probabilistic characteristics of the operation of the access network. The proposed methodology does not correspond to the objectives of the study in solving the problems of carrying out operational calculations of the performance indicators of special-purpose ITN.

Analyzed scientific sources do not take into account the peculiarities of the functioning of special-purpose ITN, the methods for calculating the characteristics of ITN are of a research or applied nature are based on complex mathematical calculations, which makes it difficult or impossible to use them in operational calculations, are repeatedly performed in the process of communication planning.

\section{Rresearch Methodology}

To propose a methodological basis for the operational calculation of the key indicator "availability" of ITN of the tactical control level and to develop a method of operational calculation that would allow officials of communications control bodies to make high-quality operational calculations of network performance indicators in a short time, taking into account the specifics of their purpose and construction.

The methodological basis of the study is the practical side of solving the problem of calculating the construction of the optimal version of the network of access to the telecommunication network of the tactical control link.

Research methodology consists in:

- the formulation of the goal and objectives of the operational planning of options for creating an access 
network

- determining the optimal option for creating an access network by synthesizing alternative structures on the basis of calculations of its indicators with the justification of evaluation criteria for property indicators;

- $\quad$ selection of techniques and means that have shown the best result.

The research methodology has the following structure:

- characteristics of the activity: features, principles, conditions and norms for the functioning of a special purpose ITN;

- logical structure of research: object $\rightarrow$ subject of research $\rightarrow$ means $\rightarrow$ methods $\rightarrow$ problem solving $\rightarrow$ result of activity;

- Methods and techniques for solving problems.

\section{Experimental Setup}

Based on the existing views in the tactical level of control, it is advisable to create a field digital information and telecommunication system, consisting of the main and mutually complementary subsystems: narrowband tactical radio, broadband subscriber radio access and broadband wire access, built on the basis of packet communication technology.

Modern multiservice information and telecommunication networks of the tactical control level are heterogeneous networks, have a hierarchical structure and contain:

- access network;

- $\quad$ access aggregation network;

- backbone segment.

The backbone segment connects the edge switches with communication channels, which provide the connection of the access aggregation network to the backbone segment.

The access aggregation network includes aggregation nodes and access nodes.

Network subscribers are connected to the access nodes using a certain topology.

A variant of the information and telecommunications network of the tactical control link is shown in Fig. 1.

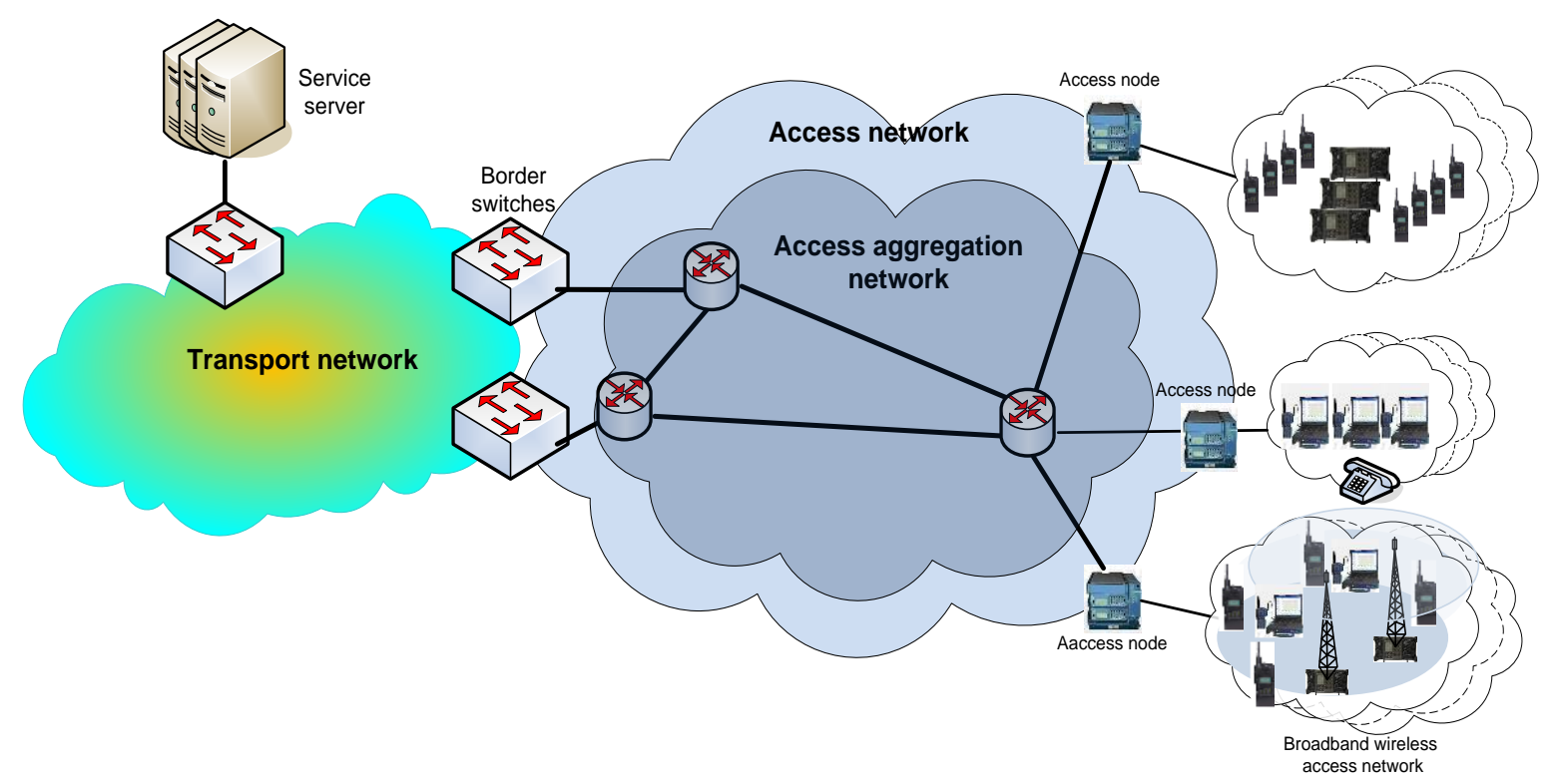

Fig.1. Option information and telecommunications network of tactical control level

An access node is a technological system that ensures the implementation of access network protocols when interacting with subscriber units, public network protocols when working with a switching node, as well as mutual conversion of these protocols and data flow control in the subscriber access system.

Access networks are the lowest level of the telecommunication network hierarchy and represent a set of access devices, communication lines with it and subscriber lines ("last mile") and is the most complex element of information and telecommunication networks.

Access networks, like telecommunication networks in general, can consist of several layers. Switches installed in the lower-level nodes multiplex the information coming through multiple subscriber channels (subscriber termination, 
local loop) and transmit it to the upper-level switches, so that they, in turn, transmit it to the trunk switches. The number of access network layers depends on its size: a small access network can consist of one layer, and a large one - from two or three. The next levels carry out further concentration of traffic, collecting it and multiplexing into higher-speed channels.

An access network can be built using a family of technologies consisting of: electrical or optical Ethernet, xDSL dedicated lines, remote access technologies and wireless access technologies. The most typical functions of the access level equipment are the concentration of users with the provision of a port (connection point) to the services of information and telecommunication networks.

When searching for the optimal option for creating an access network by synthesizing alternative structures, its indicators are calculated with the justification of evaluation criteria for property indicators.

\section{Information and Telecommunications System Analysis}

Until recently, the most problematic for implementation were the requirements for the information and telecommunications system as a whole, and for its elements in particular, in terms of bandwidth ability, combat readiness, mobility and resistance.

The resistance of an information and telecommunication system is understood as a combination of three characteristics (properties): reliability, survivability and noise immunity [10]. As a consequence, in most scientific works, resistance is subjected to integral assessment. At the same time, the use of the integral category of resistance does not provide for the rejection of independent analysis and individual assessment of each characteristic using the appropriate particular indicators and criteria [5,6].

With the transition of information and telecommunication networks to functioning over the IP protocol, an equally important requirement is put forward - "accessibility".

The structure of indicators of the properties of ITS as organizational and technical systems, their relationship and place in determining the performance of ITS are shown in Fig. 2.

As the main property, taking into account its special significance for the tactical level of control, when carrying out operational calculations, it is proposed to take the indicator "accessibility" of the telecommunication network [5, 6].

At the same time, the concept of availability includes ensuring the health of resources, but is not limited to this. For example, a service may be unavailable not because it does not work in principle, but because of the heavy load created by the tasks of other users of the system.

Under the «accessibility» of an information and telecommunications system, we mean the ability of the system to ensure that the top management (operational staff) receive the information and telecommunications services they need with the required quality while maintaining their priority and methods of establishing communication between them [6].

The structure (fig. 2) makes it possible to understand that the "availability" of a telecommunications network depends on:

1. The bandwidth of the communication system;

2. The combat readiness of the communication system, which is formed on the basis of the readiness of the elements of communication systems and the readiness of the channels, on which the reliability of communication directly depends;

3. The stability of the communication system, which is formed on such characteristics as survivability, noise immunity and reliability.

Taking into account the characteristic features of planning the deployment and operation of information and telecommunication systems of the tactical control level, such as a high degree of uncertainty in the characteristics of their functioning and the lack of initial data when planning, it is advisable to use a staff assessment model of operational-tactical calculations.

To determine the availability indicator of an access network using digital transmission systems, it is advisable to introduce a generalized indicator - the bandwidth availability factor in the form:

$$
\mathrm{K}_{\text {avail }}^{\text {band abil }}=\min \left\{\frac{V_{r e q}}{V_{p l}}, 1\right\}
$$

where $V_{\text {req }}$ - required speed rate;

$V_{p l}-$ the transmission rate of the planned (have to be implemented). 


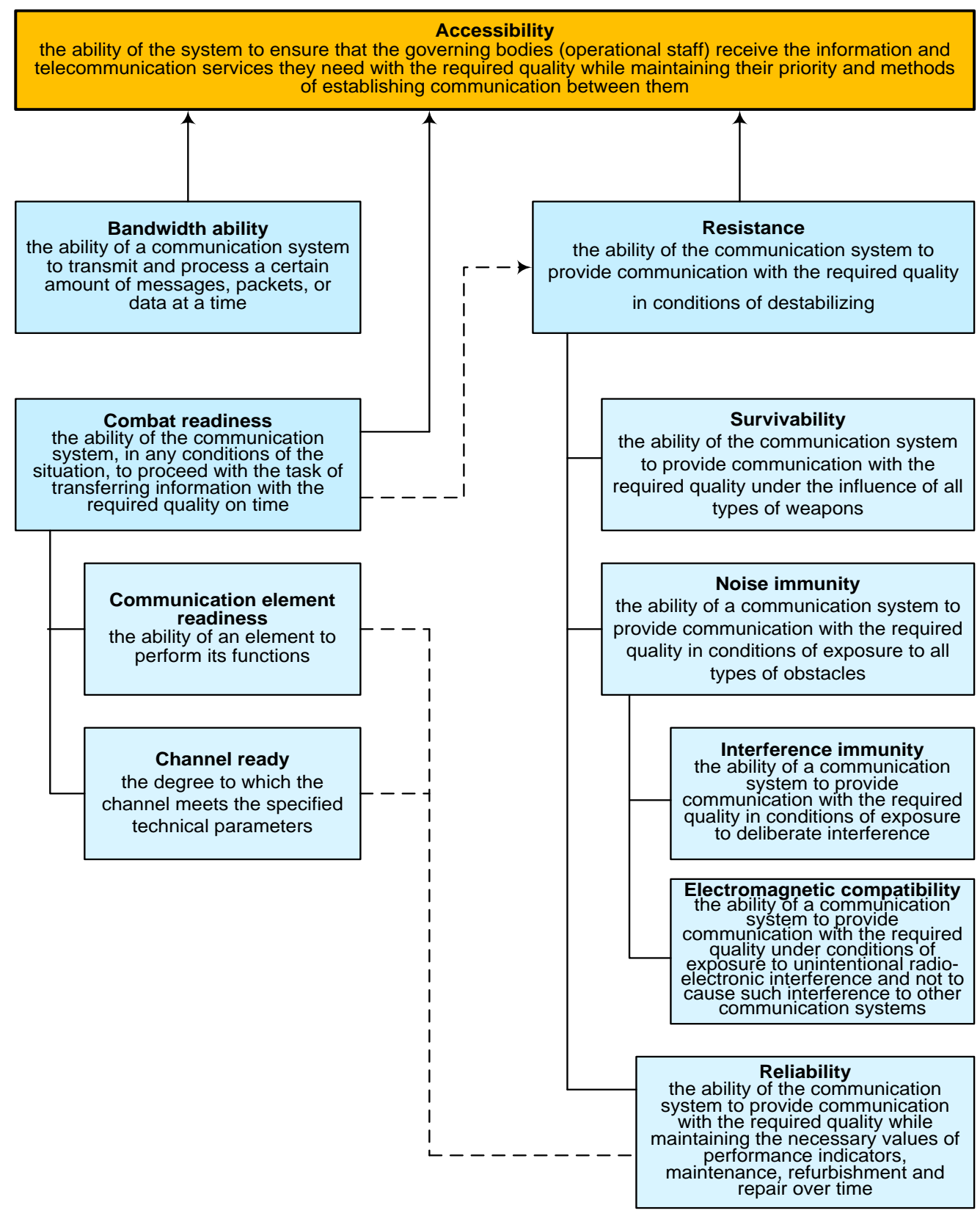

Fig.2. The structure of indicators of the properties of the information and telecommunication network

The required transmission speed $\left(V_{\text {req }}\right)$ is calculated based on the needs for information and telecommunication services for officials at control points. The need for information and telecommunication services is determined by the chief of staff of the military command body (military unit).

A typical list of telecommunications and information services to military command and control bodies at command posts, and the requirements for the data transfer rate, depend on the adopted control system and the level of development of the information and telecommunications system.

\section{Result Analysis}

It is advisable to calculate the availability of an information and telecommunications network step-by-step according to the algorithm below, taking into account the following restrictions:

- $\quad$ mathematical model of assessment - staff, assessment;

- $\quad$ user load flow is self-similar;

- the indicators "combat readiness", "stability" and "reliability" correspond to the given indicators of the system quality and are deterministic;

- $\quad$ access to the services provided to the subscriber is possible in the case of guaranteed transmission of 
information flows between the network subscriber and the border switch;

- the volume of transmitted per unit of time to ensure the specified quality of each type of information and telecommunication services is known.

Step 1. Development of a variant of the connection scheme for subscriber devices of the access network.

Based on the input data received from the chief of staff of the military control body (unit) and the communication order, after clarifying the problem, it is necessary to develop a connection scheme for subscriber devices of the access network.

The diagram must reflect:

- $\quad$ the number of users, dividing them by segments;

- $\quad$ way of connecting users;

- $\quad$ types of connecting lines;

- length of connecting lines;

- $\quad$ types of interfaces at inclusion points.

A variant of the connection diagram for subscriber devices is shown in Fig. 3.

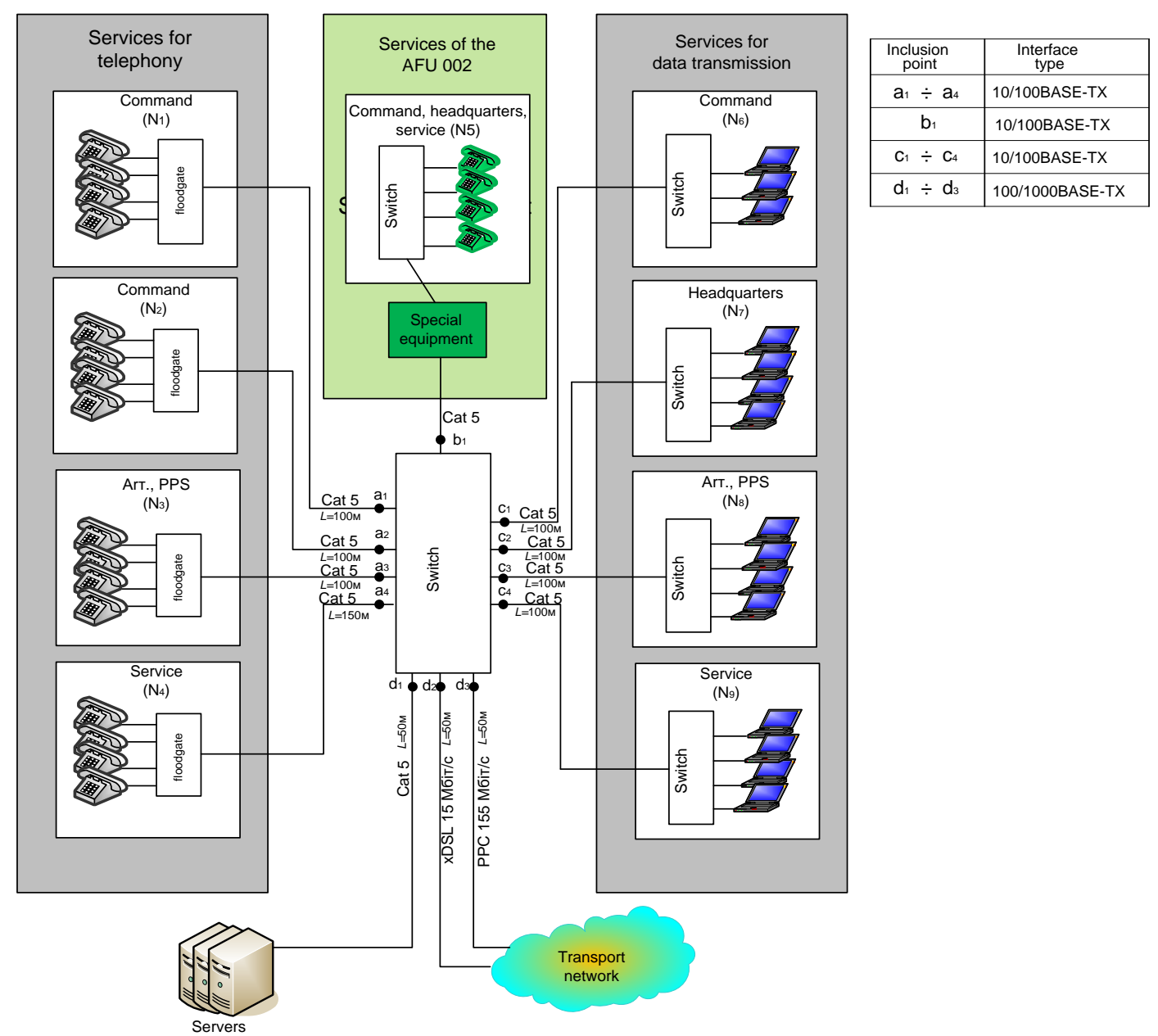

Fig.3. Connection diagram of subscriber devices

Step 2. Structuring groups of users (subscriber devices) by types of services.

In order to structure the settlements of network users with packet switching, it is advisable to conditionally divide them into two groups: users of telephone communications only and users of data transmission services, dividing them into segments $(\mathrm{Ni})$.

For both groups of users, you should specify common initial data:

$N_{i}$ - number of segments served by the access system;

$n_{j}-$ number of users in a segment;

$V_{i}$ - transmission rate for a specific type of service, Kbit/s.

Step 3. Calculation of network performance by access network segments. 
The transmission rate in telephone segments is determined by the coding rate, depending on the type of vocoder selected and the coding mode.

The minimum speed in the data transmission segment is determined by the type of each service.

The performance (transmission rate) in each network segment is calculated using the formula:

$$
C_{i}=V_{i} n_{j}
$$

where $C_{i}$ - network segment performance;

$V_{i}$ - transmission rate for a specific type of service;

$n_{j}$ - number of subscribers (users) in the segment.

Step 4. Determination of the total information transfer rate at the points of inclusion in the transport network.

The total total speed of information transfer at the points of inclusion in the transport network (at the input of the network border switch) is determined by the formula:

$$
V_{\Sigma}=\sum_{i=1}^{N} C_{i}
$$

For real networks, the important performance metric is network utilization, which is a percentage of the total bandwidth (not divided among individual users). It takes into account the internal destabilizing factors that affect network performance.

For busy Ethernet systems, it is believed that $30 \%$ is an acceptable network utilization rate. [11,12]. This value corresponds to the absence of long-term network downtime and provides sufficient headroom in the event of a peak increase in load. If the network utilization rate is $80 \%$ or more, then this indicates that the resources are almost completely used (at the present time) and leaves no reserve for the future use of the network with the established quality indicators or its further development.

Step 5. Determination of the required transmission speed in the network

To determine the required speed of information transmission in the network, it is necessary to enter the coefficient of the utilization of the network resource $(\mathrm{k})$, taking into account the delay in access to the data transmission medium. For a family of technologies Ethernet $k=0,4$ [11-13].

Then the speed of information transfer, taking into account internal destabilizing factors (collisions and the magnitude of delays in access to the data transmission medium), will be determined by the formula:

$$
V_{r e q}=V_{\Sigma} \mathrm{k}
$$

where $V_{\text {req }}$ - required transmission speed;

$k$ - network resource utilization ratio.

Step 6. Determination of the indicator of the availability of the information and telecommunication network.

Calculate the bandwidth availability ratio using the formula:

$$
K_{\text {avail }}^{\text {band abil }}=\frac{V_{\text {req }}}{V_{p l}}
$$

where $V_{\text {req }}$ - required transmission speed;

$V_{p l}-$ the transmission rate of the planned (to be realized).

If the value $K_{\text {avail }}^{\text {band abil }}$ in magnitude exceeds 1 , then the reserve of information capabilities of the network is calculated by using the expression:

$$
Q_{\text {res }}=K_{\text {avail }}^{\text {band abil }}-1
$$

The criterion for calculating the network bandwidth ability is the expression:

$$
V_{\text {rate }}<V_{p l}
$$

Step 7. Conclusion on the validity of the decision to choose a variant of the ITN structure.

Based on the analysis of the results that will be obtained in the calculations, a conclusion is made about the validity of the decision made to choose the option for the structure of the information and telecommunication network in accordance with a certain indicator of network availability $K_{\text {avail }}^{\text {band abil }}$.

In case of inconsistency of indicators $K_{\text {avail }}^{\text {band }}$, it is calculated that a new version of the access network architecture is being developed, or restrictions are introduced on the number of users and their access to network services. 
Compared with the current level of knowledge in the field of calculations in the assessment of ITN, given in the analysis of recent publications, this study and the presented calculation methodology takes into account the peculiarities of the functioning of special-purpose networks and reflects the peculiarities of solving the problems of performing operational calculations.

All operations to obtain an indicator of the availability of ITN and the load characteristics of the access network can be calculated by simple means of computer technology using the minimum set of initial data obtained when setting the problem for planning ITS and the technical characteristics of telecommunication equipment [14-16].

It is expected that the results of the study will be implemented in practice after the implementation of the methodology in the communications management bodies of the tactical level of control, its testing and evaluation with the aim of its implementation in practice.

\section{Conclusion}

Based on the analysis of selected sources of information and requirements for planning information and telecommunication systems in the tactical level of management, an approach to the operational calculation of the indicator of the functioning of access networks according to the criterion "accessibility", which characterizes the quality of subscriber service, is proposed.

The developed methodology provides an opportunity to assess the effectiveness of the communication system based on its most essential properties in terms of availability and object stability. At the same time, the availability indicator is associated with the integral stability indicator, allows a comprehensive assessment of the access network and, accordingly, make an informed decision in its construction.

The obtained assessment of the operational calculation of the indicator of the functioning of information and telecommunication networks according to the criterion - "availability" is approximate, however, it will allow officials of the communication management bodies to plan in a short time and gain confidence in their actions and the results to be achieved during planning.

All operations to obtain an indicator of the availability of the information and telecommunication network and the load characteristics of the access network can be calculated by simple computer technology.

The developed technique makes it possible to obtain the values of the bandwidth availability of information and telecommunication networks when transmitting a given amount of information, and, consequently, an assessment of the availability of an information and telecommunication network.

The proposed method of calculating the "accessibility " indicator can be used to create new and improve existing information and telecommunication networks, built using packet switching technology.

\section{Reference}

[1] Recommendation ITU-T Y.1540, 'Internet Protocol Data Communication Service - IP packet transfer and availability performance parameters', 2016. [Online]. Available: http://www.itu.int/rec/dologin_pub/id=T-REC-Y.1540-2, [Accessed 10 October 2020].

[2] Yanovsky G.G., 'Quality of Service in IP Networks’ Communication Herald, №1, 2008, pp 82-98.

[3] Report ITU -R F.2058, "Design Methods Applicable to Fixed Broadband Wireless Access Systems That Transport Internet Protocol Packets or Asynchronous Transfer Mode Cells', 2006. [Online]. Available: http://www.itu.int > rep > R-REP-F.20582006-PDF-R.pdf. [Accessed 10 October 2020].

[4] Pylinsky M.V., 'To the Assessment of Characteristics that Reflect the Features of the Functioning of the Military Communications System' Technical science, Edit 12, 2018, pp 384-389.

[5] Kalinin M.G., Pylinsky M.V., 'Methodology for Assessing the Effectiveness of the Communication System of a Combinedarms Formation according to the Generalized Indicator of Availability', Bulletin of the Military Academy of the Republic of Belarus, № 1 (54), 2017.

[6] Ageev D.V., 'Designing an Access Network for a Multiservice Telecommunications System that Maximizes the Network Operator's Profit' Bulletin of Kharkiv National University of Radio Electronics, №926, pp, 5-10, 2010.

[7] Ageev D.V., 'Designing an Access Network of a Multiservice Telecommunications System Using a Multilayer Graph', Information processing systems, Edit.8, 2010, pp 2-8.

[8] Korkin A.G., 'Estimation of the Throughput of the Radio Access System When Implementing a Hybrid Automatic Netransmission Request' Science and technology journal Information space, № 1, 2011, pp 30-35.

[9] Golubintsev A.V., 'Calculation of the Throughput of Special Purpose Wireless Access Systems', Journal of Science Intensive Technologies in Space Research of the Earth, №3, 2012, pp 4-7.

[10] Military Communications, 'State standard of Ukraine DSTU-B 3265-95', (Active from 1997.01.01). [Online]. Available: http://www.nrjetix.com/fileadmin/doc/publications/additional_info/DSTU_V_3265-1995_Military__ communications.pdf. [Accessed 10 October 2020].

[11] Goldstein B.S., Pinchuk A.V., Sukhovitsky A.L. 'IP Telephony', M.: Radio and communication, pp 1-336, 2001.

[12] Davydov A.E., Smirnov P.I., Paramonov A.I., 'Section Switched Communication Networks Calculation of Communication Network Parameters and Traffic Analysis', Saint Petersburg State University of Information Technologies, Mechanics and Optics, 2016, pp 1-47.

[13] Z. Hu, R. Odarchenko, S. Gnatyuk, M. Zaliskyi, A. Chaplits, S. Bondar, V. Borovik, Statistical Techniques for Detecting 
Cyberattacks on Computer Networks Based on an Analysis of Abnormal Traffic Behavior, International Journal of Computer Network and Information Security (IJCNIS), Vol.12, No.6, pp. 1-13, 2020.

[14] M. Sakr, W. Atwa, A. Keshk, Genetic-based Summarization for Local Outlier Detection in Data Stream, International Journal of Intelligent Systems and Applications (IJISA), Vol.13, No.1, pp. 58-68, 2021.

[15] Z. Hu, I. Tereikovskyi, D. Chernyshev, L. Tereikovska, O. Tereikovskyi, D. Wang, Procedure for Processing Biometric Parameters Based on Wavelet Transformations, International Journal of Modern Education and Computer Science (IJMECS), Vol.13, No.2, pp. 11-22, 2021.

[16] S. Ndichu, S. McOyowo, H. Okoyo et al, A Remote Access Security Model based on Vulnerability Management, International Journal of Information Technology and Computer Science (IJITCS), Vol.12, No.5, pp. 38-51, 2020.

\section{Authors' Profiles}

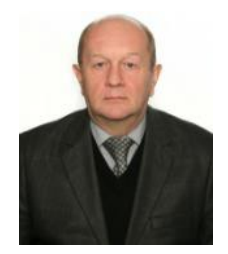

Leonid Bondarenko born in Gomel (Belarus) on 19 Oct., 1950, Senior Researcher of the Scientific Center for Communications and Informatization V.I.T.I. Rreceived a degree in telecommunications engineer in KVVIS. He has 20 years of experience in the operation of telecommunication nodes and 12 years of scientific experience. Research interests are construction and operation of information and telecommunication networks. The total number of scientific publications is 29 .

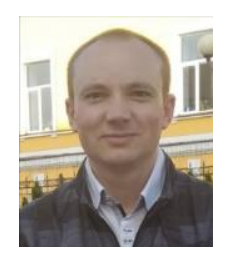

Dr. Mykola Masesov is the head of the Scientific center of the Military Institute of Telecommunications and Information Technologies Named after the Heroes of Krut. He was born in Ukraine in May 1980. He is Ph.D. in Information Technologies since 2008. He has over 15 years of experience in teaching and research positions. The range of interests includes telecommunications and information systems. The total number of publications is over 150 .

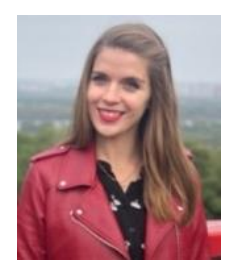

Katerina Buzayeva is a research scientist in the Military Institute of Telecommunications and Informatization, Kyiv, Ukraine. She has been working on this position for 5 years. More than 10 research publications. ggggggryui@gmail.com

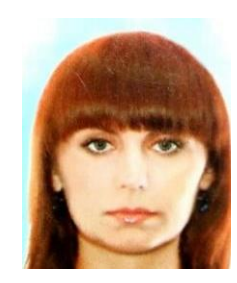

Iryna Hurzhii - Senior Researcher of the Scientific Center for Communications and Informatization of the Military Institute of Telecommunications and Informatization named after Heroes Krut. She has more than 10 years of scientific work experience, her research interests are communications, telecommunications, digital signal processing. Author of over 15 scientific publications.

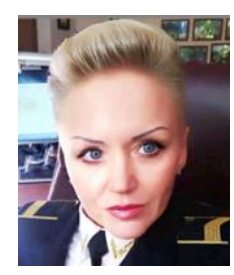

Dr. Oksana Ilienko born in Kyiv on 16th Apr. 1974. Professor at the Military Institute of Telecommunications and Information Technologies Named after the Heroes of Krut. Her last researches interests include are devoted to the impact of cyber threats on global economic markets. She has 17 years of experience in teaching and 10 years of experience on position of telecommunications engineer in Ministry of Defense of Ukraine. Total numbers of research publications are 123. 


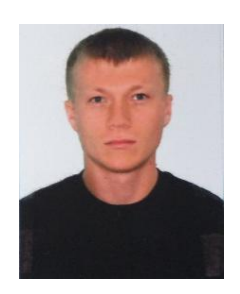

Viktor Gnatyuk is the Deputy Director of the Institute of Innovative Educational Technologies of the National Aviation University. He was born in Khmelnytsky region in July 1990. Candidate of Technical Sciences since 2017. He has more than 60 scientific publications, including collective monographs, articles in foreign publications, articles in professional publications of Ukraine.

How to cite this paper: Leonid Bondarenko, Mykola Masesov, Katerina Buzayeva, Iryna Hurzhii, Oksana Ilienko, Viktor Gnatyuk, "Method of Calculation the Accessibility of the Information and Telecommunication Networks of the Tactical Link of Management", International Journal of Computer Network and Information Security(IJCNIS), Vol.13, No.5, pp.68-78, 2021. DOI: 10.5815/ijcnis.2021.05.06 